\title{
Calculating Resonance Angle for Surface Plasmon Resonance Activation on Different Metals
}

Dang Nguyen

University of South Florida, dang3@usf.edu

Advisors:

Fernando Burgos, Mathematics and Statistics

Dmitri Voronine, Mathematics and Statistics

Physics

Problem Suggested By: Dmitri Voronine

Field of Study for Problem Suggester: Physics

Follow this and additional works at: https://digitalcommons.usf.edu/ujmm

Part of the Mathematics Commons

UJMM is an open access journal, free to authors and readers, and relies on your support:

Donate Now

\section{Recommended Citation}

Nguyen, Dang (2020) "Calculating Resonance Angle for Surface Plasmon Resonance Activation on Different Metals," Undergraduate Journal of Mathematical Modeling: One + Two: Vol. 11: Iss. 1, Article 8. DOI: https://doi.org/10.5038/2326-3652.11.1.4927

Available at: https://digitalcommons.usf.edu/ujmm/vol11/iss1/8 


\title{
Calculating Resonance Angle for Surface Plasmon Resonance Activation on Different Metals
}

\begin{abstract}
Surface plasmon resonance (SPR) is the technique that has been used in many fields including biomedical science, optic, biosensing, photothermal plasmon and medicine. With the help of Kretschmann configuration, the setup allows to excite the electrons located around the metal which results in electron oscillation, also known as the plasmonic effect. However, SPR requires many factors in order to be activated. This paper approaches and analyzes two crucial features, the incident angle of incoming light and the effect of permittivity that different metals have in order to determine the sufficient value for the plasmon to exist. Different metals including gold, silver and iron are used with the dielectric air and water in order to figure out the most applicable metal with its resonance angle.
\end{abstract}

\section{Keywords}

surface plasmon resonance, resonance angle, index of refraction

Creative Commons License

(c) (i) (9)

This work is licensed under a Creative Commons Attribution-Noncommercial-Share Alike 4.0 License. 


\section{PROBLEM STATEMENT}

Calculating the proper incident angle that the light ray needs to be shined to excite the electrons and produce plasmonic effect using the setup of Kretschmann configuration for surface plasmon resonance. In addition, examining factors that could potentially affect the incident angle such as the material of metal.

\section{MOTIVATION}

As science gets more advanced, many concepts and techniques are no longer limited in their scope. The origin of surface plasmon resonance is used to be an optical technique that detects molecular interactions. However, as time goes by, it was developed and utilized in many crucial fields such as sensing and medicine. For instance, by replacing the bulk metal with nanoparticles, the photothermal effect is enhanced dramatically which produces extensive temperature that could potentially be used to eliminate cancer cells. With the same concept, surface plasmon resonance also can be used for bacteria inactivation and serve as a potential candidate for the treatment of hereditary diseases such as cystic fibrosis ${ }^{1}$. SPR provides tremendous benefits to science and potentially has a wide range of applications in daily life. Therefore, it is essential to understand the mechanism behind the method and its condition in order to exist.

\section{MATHEMATICAL DESCRIPTION AND SOLUTION APPROACH}

\section{A) Plasmon Mechanism}

Inside a conductor, negatively charged electrons exist which move freely in the environment. When an illuminator such as sunlight or laser is applied to the conductor and starts transferring energy, the movement of the free electrons gets affected by the positive ion background. The attraction combined with the electrostatic repulsive force on free electrons creates a phenomenon of delocalized electron oscillation ${ }^{2}$. The interaction between the incident light and interface causes the photons to be absorbed and energy is transferred into electrons resulting in electrons excitation inside the metal. Electromagnetic waves are exerted along the surface and therefore they produce a strong concentration of electromagnetic field. As a result, the process generates heat and enhances local temperature which can be used for sterilization or antibacterial treatment. 


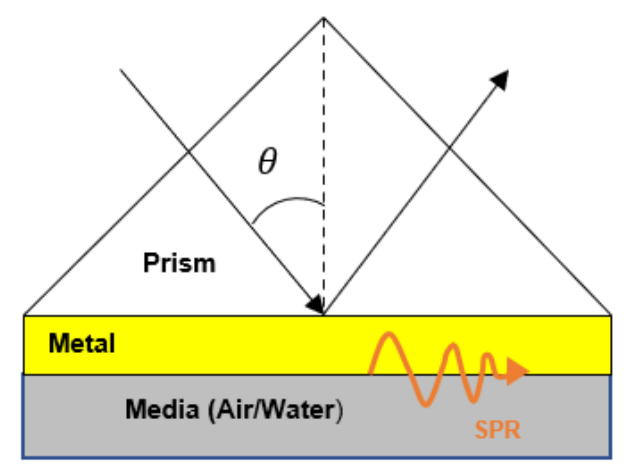

Figure 1: Kretschmann configuration producing surface plasmon resonance including 3 parts: prism, metal and media. Light source is shined with the resonance angle to cause electron oscillation.

As the light source passes through the prism that has the surface coated with a thin film of metal, a specific incident angle is needed called the resonance angle where the energy of light is absorbed causing the electrons to oscillate, or surface plasmon resonance (SPR). At that point, the incoming light photons have the same momentum with the momentum of the surface plasmons. However, with a higher or lower degree of incident angle, the resonance would not be generated and hence resulting in the absence of the plasmonic effect. There are many factors that impact the process of determining the appropriate resonant angle including the material for the metal film, refractive index of the media, wavelength of incoming light source and temperature of the surrounding area ${ }^{2}$.

To be able to transfer the energy from photons and turn into plasmons, the resonance angle needs to exert the reflection ray within total internal reflection (TIR) area without exceeding the critical angle. By applying Snell's Law with given refractive index for different metals and the prism, it is possible with the help of this formula to calculate the range of angle to produce SPR:

$$
\frac{n_{1}}{n_{2}}=\frac{\sin \left(\theta_{2}\right)}{\sin \left(\theta_{1}\right)}
$$

\section{I) $\underline{\text { Relation between SPR and incident angle }}$}

The medium that light passes through to transfer from the prism to metal has a lower index of refraction (n) which causes the transmission to be smaller than the incident angle. Moreover, it is known that the shorter the wavelength, the more energy it provides, therefore, the wavelength also impacts the refraction index. According to Pedrotti ${ }^{3}$, we have:

\begin{tabular}{|c|c|c|c|c|c|}
\hline Color & Red & Yellow & Green & Blue & Violet \\
\hline$\lambda(\mathrm{nm})$ & 640 & 589 & 509 & 486 & 434 \\
\hline $\mathrm{n}$ & 1.50917 & 1.51124 & 1.51534 & 1.51690 & 1.52136 \\
\hline
\end{tabular}

Table 1: Index of refraction of Borosilicate Crown prism with different wavelength of incoming light 
Including the effect of dimensions such as thickness and length of the metal on the incident angle would overcomplicate the problem, therefore we neglect those factors as well as assume that the experiment is performed at room temperature. In addition, each metal has its unique properties and index of refraction. However, the value is complex and is described as the formula below, in which $n_{0}$ is known as the ordinary refractive index and $\kappa$ is extinction coefficient:

$$
n=n_{0}+i \kappa
$$

The 3 metals that combine effectively to produce SPR are gold ( $\mathrm{Au})$, silver $(\mathrm{Ag})$ and iron $(\mathrm{Fe})$. Each has its own strengths and weaknesses. For example, gold is extremely resistant to oxidation when the media is in the liquid state. Meanwhile, iron is abundant in nature which can drastically reduce the cost of production. By using Refractive Index Database analysis ${ }^{4}$, the index of refraction for metals is observed. In addition, 5 standard wavelengths that are similar to incoming light rays through the prism are used for consistency.

- Gold

\begin{tabular}{|c|c|}
\hline$\lambda(\mu m)$ & $n_{\text {complex }}$ \\
\hline 0.434 & 1.4380 \\
\hline 0.486 & 1.1491 \\
\hline 0.509 & 0.8199 \\
\hline 0.589 & 0.2741 \\
\hline 0.640 & 0.1719 \\
\hline
\end{tabular}

Table 2: Different wavelengths of light shined on gold and its correlated index of refraction

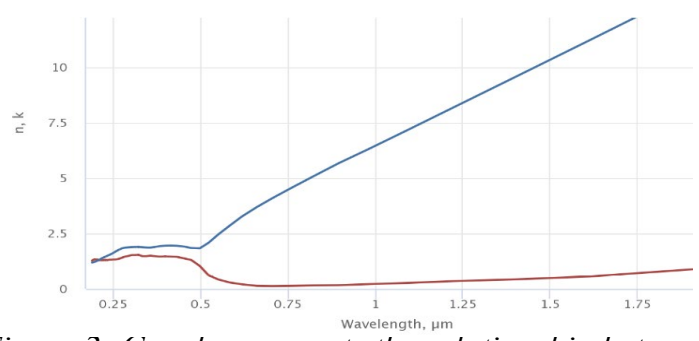

Figure 2: Graph represents the relationship between the wavelength of incoming light and index of refraction on gold

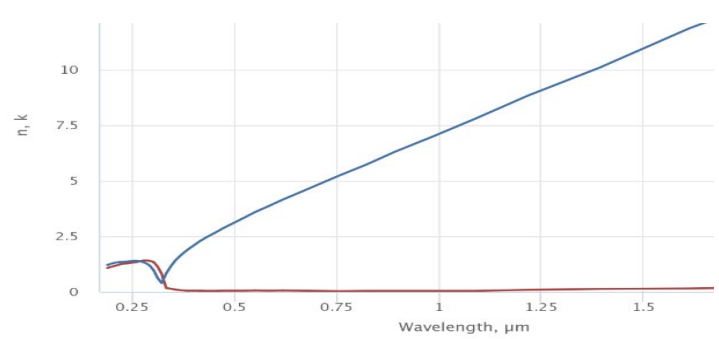

Figure 3: Graph represents the relationship between the wavelength of incoming light and index of refraction on silver 
- Iron

\begin{tabular}{|c|c|}
\hline$\lambda(\mu m)$ & $n_{\text {complex }}$ \\
\hline 0.434 & 2.4965 \\
\hline 0.486 & 2.7120 \\
\hline 0.509 & 2.8024 \\
\hline 0.589 & 2.9280 \\
\hline 0.640 & 2.9019 \\
\hline
\end{tabular}

Table 4: Different wavelengths of light shined on iron and its correlated index of refraction

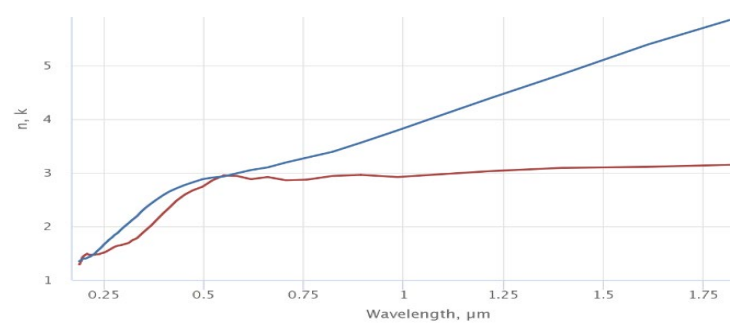

Figure 4: Graph represents the relationship between the wavelength of incoming light and index of refraction on iron

The values of wavelength are recorded in micrometers, which needs to be converted into nanometer $(1 \mu \mathrm{m}=1000 \mathrm{~nm})$. Since the index of refraction for the prism with different wavelength is close enough to each other, the average of the 5 can be used as an approximation:

$$
\begin{gathered}
\bar{n}=1.50917+1.51124+1.51534+1.56190+1.52136 \\
\bar{n}_{\text {prism }}=1.5148
\end{gathered}
$$

The formula $n_{1} \sin \left(\theta_{1}\right)=n_{2} \sin \left(\theta_{2}\right)$ in which having $n_{1}$ as $\bar{n}_{\text {prism }}, n_{2}$ is $n_{\text {complex }}$ for gold, silver and aluminum, $\theta_{1}$ is the incident angle that is restricted to produce SPR and $\theta_{2}$ is transmission angle. With gold and silver in which the refractive index is lower compared to the prism, therefore $\theta_{2}>\theta_{1}$ at all time. When $\theta_{2}$ reaches 90 degree and perpendicular to the normal line, the incident angle $\theta_{1}$ is at critical angle. As the result, the area from 0 degree to the critical angle is called $\beta$ and could cause electron oscillation producing plasmonic effect.

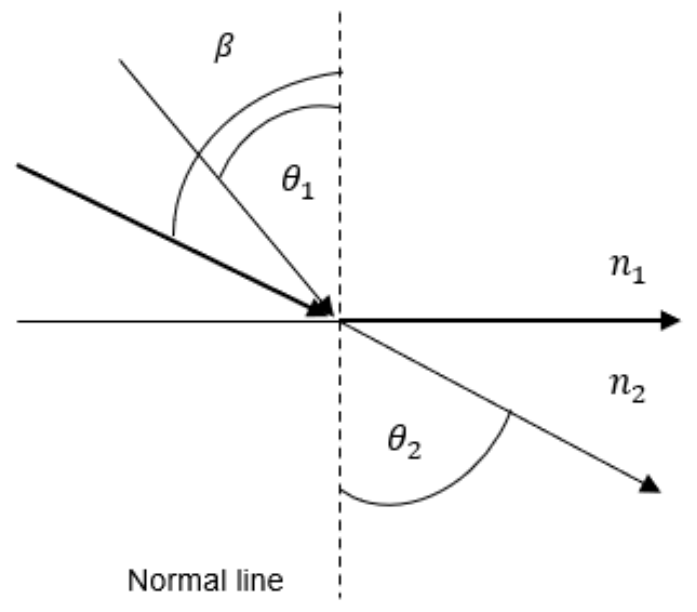

Figure 5: The image illustrates the incident light ray passing through gold/silver and is bent due to the difference in refraction index, $n_{1}>n_{2} . \beta$ represents the area from initial angle to critical angle that produce $S P R$

To calculate $\beta$, we know that $\theta_{2}$ is at 90 degree and critical angle depends on $n_{2}$ 
$n_{1} \sin \left(\theta_{1}\right)=n_{2} \sin \left(\theta_{2}\right) \Leftrightarrow \sin \left(\theta_{1}\right)=\frac{n_{2} \sin \left(\theta_{2}\right)}{n_{1}} \Leftrightarrow \theta_{1}=\sin ^{-1}\left(\frac{n_{2} \sin (90)}{1.5148}\right)$

\begin{tabular}{|c|c|c|}
\hline Wavelength $(\mu m)$ & $\beta_{\text {gold }}($ degree $)$ & $\beta_{\text {silver }}($ degree $)$ \\
\hline 0.434 & $0 \rightarrow 71.68$ & $0 \rightarrow 1.51$ \\
\hline 0.486 & $0 \rightarrow 49.34$ & $0 \rightarrow 1.89$ \\
\hline 0.509 & $0 \rightarrow 32.77$ & $0 \rightarrow 1.89$ \\
\hline 0.589 & $0 \rightarrow 10.42$ & $0 \rightarrow 1.96$ \\
\hline 0.640 & $0 \rightarrow 6.520$ & $0 \rightarrow 2.07$ \\
\hline
\end{tabular}

Table 5: resonance angle of gold and silver to active surface plasmon resonance

After calculation, the resonance angle for gold shows to be superior compared to silver since it provides much more flexibility with a wide range of incident angles to be able to effectively produce surface plasmon resonance (up to $71.68^{\circ}$ ). Moreover, it is noticeable that as the wavelength increases, the more shining area can produce SPR by gold but the area of silver will be narrower.

The same process is applied for iron. However, since the refractive index of iron is higher than the prism despite the incoming wavelength, the transmission angle $\theta_{2}$ will be smaller than the incident angle $\theta_{1}$. As a result, there is no limitation for critical angle and $\theta_{1}$ could reach to its maximum 90 degrees (perpendicular to the normal line). However, the initial angle cannot be at 0 degree since $\sin (0)=0$ and the light ray will be transferred straight through two mediums without activating SPR.

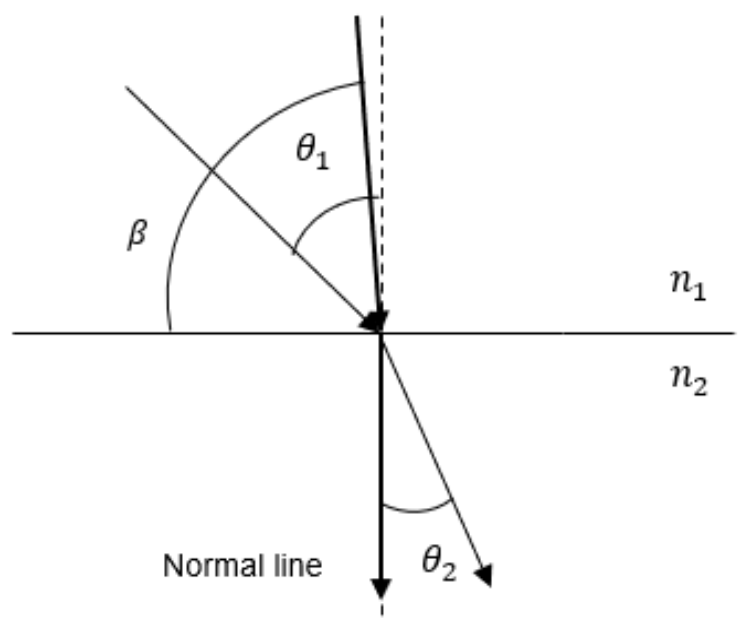

Figure 6: The image illustrates the incident light ray passing through iron and is bent due to the difference in refraction index, $n_{2}>n_{1}$. $\beta$ represents the area from initial angle to critical angle that produce SPR

Using the same calculation based on equation (1) for gold and silver, the value for resonance angle of iron can be calculated. However, the limitation for $\theta_{1}$ is set at $0.01^{\circ}$ even though it could possibly be even lower. 


\begin{tabular}{|c|c|c|}
\hline Wavelength $(\mu \mathrm{m})$ & Limited angle (degree) & $\beta_{\text {iron }}($ degree $)$ \\
\hline 0.434 & $0 \rightarrow 0.0165$ & $0.0165 \rightarrow 90$ \\
\hline 0.486 & $0 \rightarrow 0.0179$ & $0.0179 \rightarrow 90$ \\
\hline 0.509 & $0 \rightarrow 0.0185$ & $0.0185 \rightarrow 90$ \\
\hline 0.589 & $0 \rightarrow 0.0193$ & $0.0193 \rightarrow 90$ \\
\hline 0.640 & $0 \rightarrow 0.0192$ & $0.0192 \rightarrow 90$ \\
\hline
\end{tabular}

Table 7: resonance angle of iron to active surface plasmon resonance

Surprisingly, the resonance angle $(\beta)$ for iron is extremely broad in that almost all of the incoming light can transfer its energy to electrons and that makes iron a potential candidate for surface plasmon activation:

The light source with the specific angle on the prism at specific wavelength resulting in activation of SPR. Different metals show different ranges for proper incident angles, which prove that some metals are more applicable than others.

\section{II) Relation between SPR and permittivity of metal}

The resonance angle is not the only factor that could potentially affect the production of SPR but the characteristics of the metal itself such as the permittivity, $\varepsilon$, and permeability, $\mu$, also serve an important role. Previous experiment was examining the incoming angle that could produce plasmon, which is the interaction between the prism and metal film. In this case, we shall analyze the light ray after passing through the first barrier and arrive at the interface of the metal film and the dielectric, in which the media could either be water, air or simply a vacuum. Let $\varepsilon_{1}$ be the permittivity for the metal and $\varepsilon_{2}$ for dielectric.

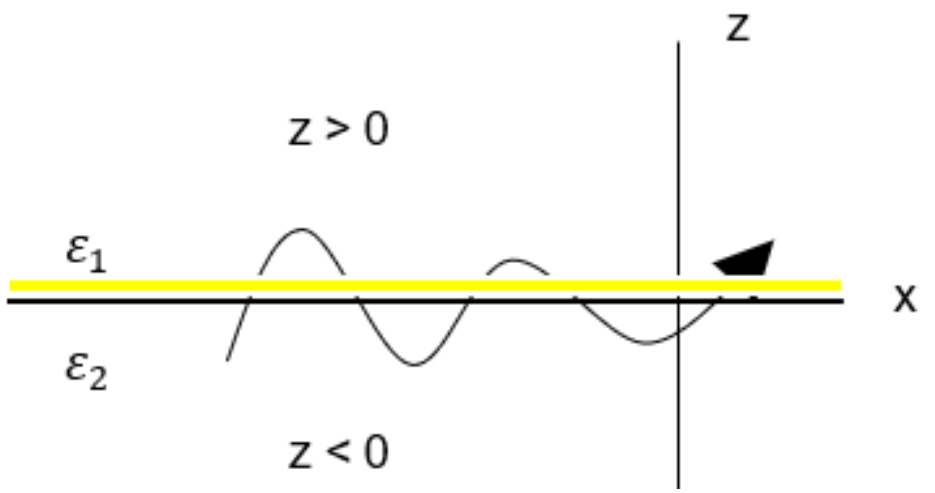

Figure 8: SPR created between the metal film and dielectric with $\varepsilon_{1}$ as the permittivity of gold / silver / iron and $\varepsilon_{2}$ as the permittivity of air / water / vacuum

Since light ray has the characteristics of a wave, it propagates around the $\mathrm{x}$-axis between $0 \square+\mathrm{z}$ and $0 \rightarrow-z$, therefore we have the condition for existence of electric field $E_{1}$ and $E_{2}$.

$$
\mathrm{z}>0: \quad E_{1}=H_{1} * \frac{1}{\varepsilon_{1} i \omega}\left(-\alpha_{1} \hat{x}-i k_{x} \hat{z}\right) * e^{i\left(k_{x} x-\omega t\right)} * e^{-\alpha_{1} z}
$$




$$
\mathrm{z}<0: \quad E_{2}=H_{2} * \frac{1}{\varepsilon_{2} i \omega}\left(\alpha_{2} \hat{x}-i k_{x} \hat{z}\right) * e^{i\left(k_{x} x-\omega t\right)} * e^{\alpha_{2} z}
$$

To be able to excite surface plasmon, 2 electric fields need to be parallel with each other at $\mathrm{z}=0$ along the x-axis as well as magnetic field $H_{1}$ and $H_{2}$. As the result, the first condition for surface plasmon resonance to exist is $H_{1}=H_{2}$

$$
\begin{aligned}
& \Leftrightarrow H_{1} * \frac{1}{\varepsilon_{1} i \omega}\left(-\alpha_{1} \hat{x}-i k_{x} \hat{z}\right) * e^{-\alpha_{1} z}=H_{2} * \frac{1}{\varepsilon_{2} i \omega}\left(\alpha_{2} \hat{x}-i k_{x} \hat{z}\right) * e^{\alpha_{2} z} \\
& \Leftrightarrow \frac{1}{\varepsilon_{1} i \omega}\left(-\alpha_{1} \hat{x}-i k_{x} \hat{z}\right) * e^{-\alpha_{1} z}=\frac{1}{\varepsilon_{2} i \omega}\left(\alpha_{2} \hat{x}-i k_{x} \hat{z}\right) * e^{\alpha_{2} z} \\
& \Leftrightarrow \frac{1}{\varepsilon_{1}}\left(-\alpha_{1} \hat{x}\right) * e^{-\alpha_{1} * 0}=\frac{1}{\varepsilon_{2}}\left(\alpha_{2} \hat{x}\right) * e^{\alpha_{2} * 0} \\
& \Leftrightarrow \frac{-\alpha_{1}}{\varepsilon_{1}}=\frac{\alpha_{2}}{\varepsilon_{2}}
\end{aligned}
$$

The equation above shows the correlation between the permittivity $(\varepsilon)$ and near field decay constant $(\alpha)$ of the metal and dielectric substance, which is also a second existence condition that the system needs to satisfy in order to generate surface plasmon excitation. According to dispersion relation, the following equation has been proved:

$$
\alpha^{2}-k_{x}^{2}=\frac{\omega^{2}}{c^{2}} \varepsilon \mu
$$

In which $k_{x}$ represents the wave vector of light, $\omega$ is the angular frequency and $\mathrm{c}$ is the speed of light $=3 * 10^{8} \mathrm{~m} / \mathrm{s}$. However, since the motion appear in the 2 areas above and below the $\mathrm{x}$-axis, we have:

$\mathrm{z}>0$ : gold/silver/iron $\rightarrow \alpha_{1}^{2}-k_{x}^{2}=\frac{\omega^{2}}{c^{2}} \varepsilon_{1} \mu_{1} \Leftrightarrow \alpha_{1}^{2}=\frac{\omega^{2}}{c^{2}} \varepsilon_{1} \mu_{1}+k_{x}^{2}$

$\mathrm{z}<0$ : water/air $\rightarrow \alpha_{2}^{2}-k_{x}^{2}=\frac{\omega^{2}}{c^{2}} \varepsilon_{2} \mu_{2} \Leftrightarrow \alpha_{2}^{2}=\frac{\omega^{2}}{c^{2}} \varepsilon_{2} \mu_{2}+k_{x}^{2}$

$\Leftrightarrow \frac{\alpha_{1}^{2}}{\alpha_{2}^{2}}=\frac{\frac{\omega^{2}}{c^{2}} \varepsilon_{1} \mu_{1}+k_{x}^{2}}{\frac{\omega^{2}}{c^{2}} \varepsilon_{2} \mu_{2}+k_{x}^{2}}$

$(2) \Leftrightarrow-\frac{\alpha_{1}}{\varepsilon_{1}}=\frac{\alpha_{2}}{\varepsilon_{2}} \Leftrightarrow-\frac{\alpha_{1}}{\alpha_{2}}=\frac{\varepsilon_{1}}{\varepsilon_{2}} \Leftrightarrow-\left(\frac{\alpha_{1}}{\alpha_{2}}\right)^{2}=\left(\frac{\varepsilon_{1}}{\varepsilon_{2}}\right)^{2} \Leftrightarrow \frac{\alpha_{1}^{2}}{\alpha_{2}^{2}}=-\frac{\varepsilon_{1}^{2}}{\varepsilon_{2}^{2}}$

(3), (4) $\Leftrightarrow-\frac{\varepsilon_{1}^{2}}{\varepsilon_{2}^{2}}=\frac{\frac{\omega^{2}}{c^{2}} \varepsilon_{1} \mu_{1}+k_{x}^{2}}{\frac{\omega^{2}}{c^{2}} \varepsilon_{2} \mu_{2}+k_{x}^{2}} \Leftrightarrow-\left(\frac{\omega^{2}}{c^{2}} * \varepsilon_{1}^{2} \varepsilon_{2} \mu_{2}+\varepsilon_{1}^{2} * k_{x}^{2}\right)=\frac{\omega^{2}}{c^{2}} * \varepsilon_{2}^{2} \varepsilon_{1} \mu_{1}+\varepsilon_{2}^{2} * k_{x}^{2}$

$\Leftrightarrow k_{x}^{2} *\left(\varepsilon_{2}^{2}-\varepsilon_{1}^{2}\right)=\frac{\omega^{2}}{c^{2}} * \varepsilon_{1} \varepsilon_{2}\left(\varepsilon_{2} \mu_{1}-\varepsilon_{1} \mu_{2}\right) \Leftrightarrow k_{x}=\frac{\omega}{c} * \sqrt{\frac{\varepsilon_{1} \varepsilon_{2}\left(\varepsilon_{2} \mu_{1}-\varepsilon_{1} \mu_{2}\right)}{\left(\varepsilon_{2}-\varepsilon_{1}\right)\left(\varepsilon_{2}+\varepsilon_{1}\right)}}$ 


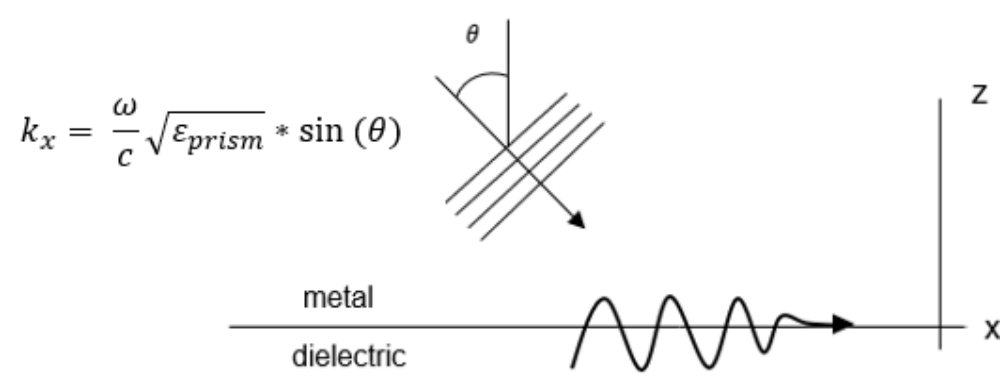

Figure 9: Wave vector of the light ray transfer from the metal through dielectric

As light comes from the prism to the metal, its wave vector can be illustrated as:

$$
k_{x}=\frac{\omega}{c} \sqrt{\varepsilon_{\text {prism }}} * \sin (\theta)
$$

Wave vector $k_{x_{\text {prism to metal }}}$ needs to be equivalent to the wave vector $k_{x_{\text {metal to dielectric }}}$ in equation (5) in order to conduct surface plasmon resonance:

$$
\begin{aligned}
& \Leftrightarrow \frac{\omega}{c} \sqrt{\varepsilon_{\text {prism }}} * \sin (\theta)=\frac{\omega}{c} * \sqrt{\frac{\varepsilon_{1} \varepsilon_{2}\left(\varepsilon_{2} \mu_{1}-\varepsilon_{1} \mu_{2}\right)}{\left(\varepsilon_{2}-\varepsilon_{1}\right)\left(\varepsilon_{2}+\varepsilon_{1}\right)}} \\
& \Leftrightarrow \sin (\theta) \sqrt{\varepsilon_{\text {prism }}}=\sqrt{\frac{\varepsilon_{1} \varepsilon_{2}\left(\varepsilon_{2} \mu_{1}-\varepsilon_{1} \mu_{2}\right)}{\left(\varepsilon_{2}-\varepsilon_{1}\right)\left(\varepsilon_{2}+\varepsilon_{1}\right)}} \Leftrightarrow \sin (\theta)=\sqrt{\frac{\varepsilon_{2}\left(\varepsilon_{2} \mu_{1}-\varepsilon_{1} \mu_{2}\right)}{\left(\varepsilon_{2}-\varepsilon_{1}\right)\left(\varepsilon_{2}+\varepsilon_{1}\right)}} \\
& \Leftrightarrow \theta=\sqrt{\frac{\varepsilon_{2}\left(\varepsilon_{2} \mu_{1}-\varepsilon_{1} \mu_{2}\right)}{\left(\varepsilon_{2}-\varepsilon_{1}\right)\left(\varepsilon_{2}+\varepsilon_{1}\right) \varepsilon_{\text {prism }}}}
\end{aligned}
$$

The incident angle equation is derived based on the relative permeability $(\mu)$ and permittivity $(\varepsilon)$ of the metal; however, each metal has different values resulting in a dissimilar effect on the system. While the permeability is relatively independent from the refractive index (n) for gold and silver (not iron), the permittivity is not. By applying Maxwell's equation, we can find the correlation among them. However, $n_{\text {complex }}$ varies based on $\lambda$ of incident light, therefore, 509 $\mathrm{nm}$ will be used as an example to receive the correlated $\mathrm{n}$ for 3 different metals.

$$
\begin{aligned}
& \rightarrow n_{\text {gold }}=0.8199 \\
& \rightarrow n_{\text {silver }}=0.0500 \\
& \rightarrow n_{\text {iron }}=2.8024
\end{aligned}
$$

We have:

$$
\nabla \times \mathrm{E}=-\frac{\partial \mathrm{B}}{\partial \mathrm{t}}(\mathrm{i}) \quad \nabla \times \mathrm{B}=\mu \varepsilon \frac{\partial \mathrm{E}}{\partial \mathrm{t}}(i i) \quad \nabla \cdot \mathrm{E}=0
$$


In which $\mathrm{E}$ is an electric field and B is the magnetic flux density. Moreover, the electric field can also be represented by the equation including the wavelength of incoming light $(\lambda)$ and the speed of light (c). To simplify the problem, the function only considers $1 \mathrm{D}$ interactions based on 2 variables, $\mathrm{x}$ and $\mathrm{t}$ :

$$
E=E_{0} \sin \left(2 \pi \frac{x-c t}{\lambda}\right)
$$

We have:

$\nabla \times \mathrm{E}=-\frac{\partial \mathrm{B}}{\partial \mathrm{t}} \Leftrightarrow \nabla \times \nabla \times \mathrm{E}=\nabla \times\left(-\frac{\partial \mathrm{B}}{\partial \mathrm{t}}\right) \Leftrightarrow \nabla^{2} \times \mathrm{E}=-\frac{\partial}{\partial \mathrm{t}}(\nabla \times \mathrm{B})$

Substitute second equation (ii) for $\nabla \times B$ in derived equation (i)

$\nabla \times(\nabla \times \mathrm{E})=-\frac{\partial}{\partial \mathrm{t}}\left(\mu \varepsilon \frac{\partial \mathrm{E}}{\partial \mathrm{t}}\right) \Leftrightarrow \nabla \times(\nabla \times \mathrm{E})=-\mu \varepsilon \frac{\partial^{2} E}{\partial \mathrm{t}^{2}}$

The notation $\nabla \times E$ represents the "curl" of an electric field, in which the curl of the curl $\nabla \times(\nabla \times E)$ has been proved that it can be illustrated in another form ${ }^{5}$

$$
\nabla \times(\nabla \times \mathrm{E})=\nabla \cdot(\nabla \cdot \mathrm{E})-\nabla^{2} E
$$

$\Leftrightarrow \nabla \cdot(\nabla \cdot \mathrm{E})-\nabla^{2} E=-\mu \varepsilon \frac{\partial^{2} E}{\partial \mathrm{t}^{2}}$

According to the third equation of Maxwell (iii) and assuming that the charge density is zero, the equation can be simplified to $\nabla^{2} E=\mu \varepsilon \frac{\partial^{2} E}{\partial t^{2}}$. The gradient symbol shows the partial derivative in three dimension:

$$
\nabla^{2} E=\frac{\partial^{2} E}{\partial x^{2}}+\frac{\partial^{2} E}{\partial y^{2}}+\frac{\partial^{2} E}{\partial z^{2}}
$$

However, we will solve for one dimension, $\mathrm{x}$, which means calculating the condition of existence for surface plasmon resonance in $\mathrm{x}$-direction only.

$$
\begin{aligned}
& \Leftrightarrow \frac{\partial^{2} E}{\partial x^{2}}=\mu \varepsilon \frac{\partial^{2} E}{\partial t^{2}} \\
& (7),(8) \Leftrightarrow \frac{\partial^{2}}{\partial x^{2}} E_{0} \sin \left(2 \pi \frac{x-c t}{\lambda}\right)=\mu \varepsilon \frac{\partial^{2}}{\partial t^{2}} E_{0} \sin \left(2 \pi \frac{x-c t}{\lambda}\right) \\
& \Leftrightarrow \frac{\partial}{\partial x}\left[\frac{2 \pi}{\lambda} E_{0} \cos \left(2 \pi \frac{x-c t}{\lambda}\right)\right]=-\mu \varepsilon \frac{\partial}{\partial t}\left[\frac{2 \pi}{\lambda} E_{0} \cos \left(2 \pi \frac{x-c t}{\lambda}\right) * c\right] \\
& \Leftrightarrow-\frac{4 \pi^{2}}{\lambda^{2}} E_{0} \sin \left(2 \pi \frac{x-c t}{\lambda}\right)=-\mu \varepsilon * \frac{4 \pi^{2}}{\lambda^{2}} E_{0} \sin \left(2 \pi \frac{x-c t}{\lambda}\right) * c^{2} \\
& \Leftrightarrow c^{2} \mu \varepsilon=1 \Leftrightarrow c=\sqrt{\frac{1}{\mu \varepsilon}}
\end{aligned}
$$


The refractive index (n) is defined as $\frac{\text { speed of light in the vacuum }}{\text { speed of light in the substance }}, n=\frac{c_{0}}{c_{\text {metal }}}=\frac{\sqrt{\left(\mu_{0} \varepsilon_{0}\right)^{-1}}}{\sqrt{\left(\mu_{\text {metal }} \varepsilon_{\text {metal }}\right)^{-1}}}$

$\Leftrightarrow n=\frac{\sqrt{\mu_{\text {metal }} \varepsilon_{\text {metal }}}}{\sqrt{\mu_{0} \varepsilon_{0}}} \Leftrightarrow n=\sqrt{\frac{\mu_{\text {metal }} \varepsilon_{\text {metal }}}{\mu_{0} \varepsilon_{0}}}$

All the needed equations are derived to calculate the resonance angle and help to analyze how the characteristic of different materials affects the production of surface plasmon resonance. The following part B calculation will divide into 2 subsections, one for gold, silver and one for iron since they have many essential differences from each other.

\section{B) Calculation}

$$
\text { (6) } \Leftrightarrow \theta=\sqrt{\frac{\varepsilon_{2}\left(\varepsilon_{2} \mu_{1}-\varepsilon_{1} \mu_{2}\right)}{\left(\varepsilon_{2}-\varepsilon_{1}\right)\left(\varepsilon_{2}+\varepsilon_{1}\right) \varepsilon_{\text {prism }}}}
$$

According to the Appendix ${ }^{7}$, the relative permeability for gold and silver $\left(\mu_{1}\right)$ is both equal to 1 as well as the permeability for air $\left(\mu_{2}\right)$. On the other hand, in liquid dielectric, the value varies based on many factors such as the temperature or fluid distribution of the environment that the light rays are passing through, which results in multiple outcomes and correlated resonance angles. To simplify the issue, standard relative permeability $1\left(N / A^{2}\right)$ will be used for water.

$$
\Leftrightarrow \theta=\sqrt{\frac{\varepsilon_{2}}{\left(\varepsilon_{2}+\varepsilon_{1}\right) \varepsilon_{\text {prism }}}}
$$

By using the wavelength of incoming light ray at $509 \mathrm{~nm}$, correlated refractive index of 3 different metals was recorded in part 1:

$$
\begin{aligned}
& n_{\text {gold }}=0.8199 \\
& n_{\text {silver }}=0.0500 \\
& n_{\text {iron }}=2.8024 \\
& n_{\text {prism }}=1.5148 \Leftrightarrow \epsilon_{r_{\text {prism }}}=2.2946
\end{aligned}
$$

\section{- $\quad$ Gold and silver}

Since gold and silver is diamagnetic material, its permeability is approximately equal to the permeability of the vacuum ${ }^{6}$, which make equation 9 showing the relationship between the refractive index $\mathrm{n}$ with $\lambda, \mu$ can be abbreviated to: 
$n=\sqrt{\frac{\varepsilon_{\text {metal }}}{\varepsilon_{0}}} \Leftrightarrow n=\sqrt{\epsilon_{r}}$ with $\epsilon_{r}$ as the relative permittivity for metal which is the same with $\varepsilon_{2}$ in equation 10. $\varepsilon_{1}$ is the dielectric that could either be air $\left(\varepsilon_{1}=1 \mathrm{~F} / \mathrm{m}\right)$ or water $\left(\varepsilon_{1}=80 \mathrm{~F} / \mathrm{m}\right)^{7}$

$$
n_{\text {gold }}=0.8199 \rightarrow \epsilon_{r_{\text {gold }}}=0.6722
$$

For air: $\theta=\sin ^{-1} \sqrt{\frac{\varepsilon_{2}}{\left(\varepsilon_{2}+\varepsilon_{1}\right) \varepsilon_{\text {prism }}}}=\sin ^{-1} \sqrt{\frac{0.6722}{(0.6722+1) * 2.2946}}=24.74^{\circ}$

For water: $\theta=\sin ^{-1} \sqrt{\frac{\varepsilon_{2}}{\left(\varepsilon_{2}+\varepsilon_{1}\right) \varepsilon_{\text {prism }}}}=\sin ^{-1} \sqrt{\frac{0.6722}{(0.6722+80) * 2.2946}}=3.45^{\circ}$

$$
n_{\text {silver }}=0.0500 \rightarrow \epsilon_{r_{\text {silver }}}=0.0025
$$

For air: $\theta=\sin ^{-1} \sqrt{\frac{\varepsilon_{2}}{\left(\varepsilon_{2}+\varepsilon_{1}\right) \varepsilon_{\text {prism }}}}=\sin ^{-1} \sqrt{\frac{0.0025}{(0.6722+1) * 2.2946}}=1.46^{\circ}$

For water: $\theta=\sqrt{\frac{\varepsilon_{2}}{\left(\varepsilon_{2}+\varepsilon_{1}\right) \varepsilon_{\text {prism }}}}=\sqrt{\frac{0.0025}{(0.6722+80) * 2.2946}}=0.21^{\circ}$

$\Rightarrow$ Recall that in Part A section $I$, the range that light can be reflected at wavelength $509 \mathrm{~nm}$ for gold is 0 to $32.77^{\circ}$ and silver is 0 to $1.89^{\circ}$. With the specific wavelength which lead to unique refractive index and therefore the permittivity of metal, gold need to be shined exactly at $24.74^{\circ}$ for air, $3.45^{\circ}$ for water and silver need to be shined exactly at $1.46^{\circ}$ for air, $0.21^{\circ}$ respectively in order to produce surface plasmon resonance.

- $\underline{\text { Iron }}$

Unlike gold, silver or other metals, relative permeability and permittivity of iron is not a constant value. As the magnetic field increases, the value rises up, reaches maximum and then drops back down again. According to the Appendix ${ }^{8}, 99.9 \%$ pure iron can have the permeability up to $350,000\left(N / A^{2}\right)$ as opposed to $1\left(N / A^{2}\right)$ of gold and silver which make it not possible for exact resonance angle identification. As a result, metals such as gold, silver and aluminum are utilized more frequently in surface plasmon resonance since they provide more consistency and effectiveness.

\section{DISCUSSION}

The purpose of this experiment is to find the proper incident angle, also known as resonance angle, that can excite the system to exert electron oscillation and produce the plasmonic effect. Three different metals, gold $(\mathrm{Au})$, silver $(\mathrm{Ag})$ and iron $(\mathrm{Fe})$, were chosen in order to examine the 
difference in characteristics and how it could potentially affect the outcome. The result shows that gold and silver are potential candidates for surface plasmon resonance due to their quantitative relative permittivity and permeability, which results in a consistent process and therefore is able to calculate and predict the angle that light ray needs to come from. On the other hand, since iron is influenced by the magnetic field, it is shown to be less compatible with SPR compared to most other metals. The experiment also examines the resonance angle for gold and silver in the air and water, which eventually turns out that in a liquid environment, the angle is much narrower the capability of producing plasmon. For gold, the light needs to come at an angle of $3.45^{\circ}$ in the water as opposed to $24.74^{\circ}$ in air environment in order to activate SPR. For silver, it is $0.21^{\circ}$ in water and $1.46^{\circ}$ in air. This happens because of the difference in refractive index and permittivity between 2 environments, causing the light ray to change the direction. However, the experiment is assumed to be performed in the ideal situation without any human or systematic error. Therefore, in real-life applications, many other factors need to be taken into consideration that could potentially affect the results.

\section{CONCLUSION AND RECOMMENDATIONS}

Surface plasmon resonance is an important technique for its countless applications in modern life, therefore, more in-depth research related to the field is needed in order to study further. The paper approaches one of the primary features, the foundation of surface plasmon resonance, which is the incident angle of the incoming light source. There are many factors that were neglected for simplicity purpose such as calculating the resonance angle of iron. Gold has shown the largest incident angle compare to silver and iron which make it more flexible for activating SPR. Even though iron seems to be less effective, it is abundant and inexpensive make iron a promising candidate for SPR technique. However, more advanced methods need to be developed in the future in order to integrate this metal with surface plasmon. Experiment on different environments that the light is shined through is also performed. The result shows that air provides a larger incident angle than water due to the difference in the index of refraction. The concept of the experiment can be applied to many related fields. For example, multiple heat generation models for surface plasmon resonance were developed in the past and if we can calculate the angle needed to produce the heat, we could possibly determine which incident angle or what type of wavelength produces the most amount of heat. Furthermore, there are also studies showing the resistance levels of bacteria and cancer cells to the intensive temperature created by SPR, therefore, surface plasmon plays an important role in the science field. For those who are interested in the project, they can analyze different types of metals that have similar properties as gold and silver to determine its resonance angle in multiple environments. 


\section{NOMENCLATURE}

\begin{tabular}{|c|c|c|}
\hline Symbol & Meaning & Unit \\
\hline $\mathrm{n}$ & Refractive index & $\mathrm{N} / \mathrm{a}$ \\
\hline $\mathrm{c}$ & Speed of light & $\mathrm{m} / \mathrm{s}$ \\
\hline$c_{0}$ & Speed of light in vacuum & $\mathrm{m} / \mathrm{s}$ \\
\hline$\lambda$ & Wavelength & $\mathrm{nm}$ \\
\hline$\alpha$ & Near field decay constant & $s^{-1}$ \\
\hline$\varepsilon$ & Permittivity & $\mathrm{F} / \mathrm{m}$ \\
\hline$\varepsilon_{0}$ & Permittivity in vacuum & $\mathrm{F} / \mathrm{m}$ \\
\hline$\varepsilon_{1}$ & Permittivity of metal film & $\mathrm{F} / \mathrm{m}$ \\
\hline$\varepsilon_{2}$ & $\begin{array}{l}\text { Permittivity of the } \\
\text { dielectric }\end{array}$ & $\mathrm{F} / \mathrm{m}$ \\
\hline$\epsilon_{r}$ & Relative permittivity & $\mathrm{F} / \mathrm{m}$ \\
\hline$\mu$ & Permeability & $N / A^{2}$ \\
\hline$\mu_{0}$ & Permeability in vacuum & $N / A^{2}$ \\
\hline B & Magnetic flux density & $\mathrm{Wb} / m^{2}$ \\
\hline $\mathrm{E}$ & Electric field & $\mathrm{V} / \mathrm{m}$ \\
\hline$\beta$ & Resonance angle & degree \\
\hline
\end{tabular}

\section{REFERENCES}

[1]: Ambardar, S.; Nguyen, D.; Binder, G.; Withers, Z.W.; Voronine, D.V. Quantum Leap from Gold and Silver to Aluminum Nanoplasmonics for Enhanced Biomedical Applications. Appl. Sci. 2020, 10, 4210.

[2]: A. K. Sharma, R. Jha and B. D. Gupta, "Fiber-Optic Sensors Based on Surface Plasmon Resonance: A Comprehensive Review," in IEEE Sensors Journal, vol. 7, no. 8, pp. 1118-1129, Aug. 2007. 
[3]: Elert, G. (n.d.). Index of Refraction of Glass, Crown. Retrieved April 23, 2020, from https://hypertextbook.com/facts/2005/JustinChe.shtml

[4]: RefractiveIndex.INFO. (n.d.). Retrieved April 23, 2020, from

https://refractiveindex.info/?shelf=main\&book=Au\&page=Johnson

[5]: Electrodynamics-The Curl of the Curl. (n.d.). Retrieved April 18, 2020, from https://www.mathpages.com/home/kmath563/kmath563.htm

[6]: The dielectric constant and the refractive index. (n.d.). Retrieved April 19, 2020, from https://www.doitpoms.ac.uk/tlplib/dielectrics/dielectric_refractive_index.php

[7]: Helhel, S., Tosun, P. D., \& Ozen, S. (n.d.). Shielding Effectiveness of Plasma Coated Multilayered Shields. doi: 10.13140/2.1.4952.5444

[8]: (Mechanical), R. C. E., (Materials), E. S., (Materials), M. B., (Mechanical), hacksaw, \& (Automotive), dgallup. (2017, August 31). Magnetic Permeability: 1008 vs 1018. Retrieved April 23, 2020, from https://www.eng-tips.com/viewthread.cfm?qid=429538

\section{APPENDIX}

\begin{tabular}{c|c|c}
\hline Material & $\sigma_{r}[\mathbf{S} / \mathrm{m}]$ & $\mu_{r}[\mathbf{H} / \mathrm{m}]$ \\
\hline Silver & 1.05 & 1 \\
\hline Copper & 1 & 1 \\
\hline Gold & 0.7 & 1 \\
\hline Stainless Steel (430) & 0.02 & 500 \\
\hline Steel (SAE 1045) & 0.1 & 1000 \\
\hline
\end{tabular}

Table 1: Relative permeability of gold and silver ${ }^{7}$ 
Nguyen: Calculating Resonance Angle for Surface Plasmon Resonance A ctivat

Table 2.2 Magnetic properties of high-permeability metals and alloys.

\begin{tabular}{|c|c|c|c|c|c|}
\hline Material & Composition (wt\%) & $\mu_{i}\left(\mu_{0}\right)$ & $\mu_{\mathrm{ma}}\left(\mu_{0}\right)$ & $H_{c}\left(\mathrm{~A} \mathrm{~m}^{-1}\right)$ & $J_{z}(\mathrm{~T})$ \\
\hline Iron & Commercial $99 \mathrm{Fe}$ & 200 & 6000 & 70 & 2.16 \\
\hline Iron & Pure $99.9 \mathrm{Fe}$ & 25000 & 350000 & 0.8 & 2.16 \\
\hline Silicon-iron & $96 \mathrm{Fe}-4 \mathrm{Si}$ & 500 & 7000 & 40 & 1.95 \\
\hline $\begin{array}{r}\text { Silicon-iron } \\
(110)[001]\end{array}$ & $97 \mathrm{Fe}-3 \mathrm{Si}$ & 9000 & 40000 & 12 & 2.01 \\
\hline $\begin{array}{l}\text { Silicon-iron } \\
\{100\}\langle 100\rangle\end{array}$ & $97 \mathrm{Fe}-3 \mathrm{Si}$ & - & 100000 & 6 & 2.01 \\
\hline Mild steel & $\begin{array}{c}\mathrm{Fe}-0.1 \% \mathrm{C}-0.1 \% \mathrm{Si} \\
-0.4 \% \mathrm{Mn}\end{array}$ & 800 & 1100 & 200 & - \\
\hline Hypernik & $50 \mathrm{Fe}-50 \mathrm{Ni}$ & 4000 & 70000 & 4 & 1.60 \\
\hline Deltamax $\{100\}\langle 100\rangle$ & $50 \mathrm{Fe}-50 \mathrm{Ni}$ & 500 & 200000 & 16 & 1.55 \\
\hline Isoperm $\{100\}\langle 100\rangle$ & $50 \mathrm{Fe}-50 \mathrm{Ni}$ & 90 & 100 & 480 & 1.60 \\
\hline 78 Permalloy & $78 \mathrm{Ni}-22 \mathrm{Fe}$ & 4000 & 100000 & 4 & 1.05 \\
\hline Supermalloy & $79 \mathrm{Ni}-16 \mathrm{Fe}-5 \mathrm{Mo}$ & 100000 & 1000000 & 0.15 & 0.79 \\
\hline Mumetal & $77 \mathrm{Ni}-16 \mathrm{Fe}-5 \mathrm{Cu}-2 \mathrm{Cr}$ & 20000 & 100000 & 4 & 0.75 \\
\hline Hyperco & $64 \mathrm{Fe}-35 \mathrm{Co}-0.5 \mathrm{Cr}$ & 650 & 10000 & 80 & 2.42 \\
\hline Permendur & $50 \mathrm{Fe}-50 \mathrm{Co}$ & 500 & 6000 & 160 & 2.46 \\
\hline 2V-Permendur & $49 \mathrm{Fe}-49 \mathrm{Co}-2 \mathrm{~V}$ & 800 & 4000 & 160 & 2.45 \\
\hline Supermendur & $49 \mathrm{Fe}-49 \mathrm{Co}-2 \mathrm{~V}$ & - & 60000 & 16 & 2.40 \\
\hline 25 Perminvar & $45 \mathrm{Ni}-30 \mathrm{Fe}-25 \mathrm{Co}$ & 400 & 2000 & 100 & 1.55 \\
\hline 7 Perminvar & $70 \mathrm{Ni}-23 \mathrm{Fe}-7 \mathrm{Co}$ & 850 & 4000 & 50 & 1.25 \\
\hline $\begin{array}{l}\text { Perminvar } \\
\text { (magnetically } \\
\text { annealed) }\end{array}$ & $43 \mathrm{Ni}-34 \mathrm{Fe}-23 \mathrm{Co}$ & - & 400000 & 2.4 & 1.50 \\
\hline Alperm or Alfenol & $84 \mathrm{Fe}-16 \mathrm{Al}$ & 3000 & 55000 & 3.2 & 0.80 \\
\hline Alfer & $87 \mathrm{Fe}-13 \mathrm{Al}$ & 700 & 3700 & 53 & 1.20 \\
\hline Aluminium-iron & $96.5 \mathrm{Fe}-3.5 \mathrm{Al}$ & 500 & 19000 & 24 & 1.90 \\
\hline Sendust & $85 \mathrm{Fe}-10 \mathrm{Al}-5 \mathrm{Al}$ & 36000 & 120000 & 1.6 & 0.89 \\
\hline
\end{tabular}

Table 2: Relative permeability for $99.9 \%$ pure iron ${ }^{8}$ 